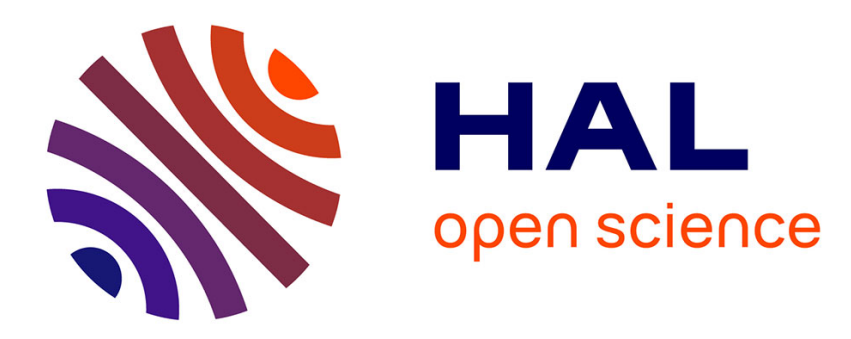

\title{
Vibration properties of a percolating cluster
}

\author{
G.S. Grest, I. Webman
}

\section{To cite this version:}

G.S. Grest, I. Webman. Vibration properties of a percolating cluster. Journal de Physique Lettres, 1984, 45 (24), pp.1155-1160. 10.1051/jphyslet:0198400450240115500 . jpa-00232463

\section{HAL Id: jpa-00232463 https://hal.science/jpa-00232463}

Submitted on 1 Jan 1984

HAL is a multi-disciplinary open access archive for the deposit and dissemination of scientific research documents, whether they are published or not. The documents may come from teaching and research institutions in France or abroad, or from public or private research centers.
L'archive ouverte pluridisciplinaire HAL, est destinée au dépôt et à la diffusion de documents scientifiques de niveau recherche, publiés ou non, émanant des établissements d'enseignement et de recherche français ou étrangers, des laboratoires publics ou privés. 
Classification

Physics Abstracts

$63.50-62.20-46.20 \mathrm{C}$

\title{
Vibration properties of a percolating cluster
}

\author{
G. S. Grest and I. Webman \\ Corporate Research Science Laboratory, Exxon Research and Engineering Company, \\ Annandale, New Jersey 08801, U.S.A.
}

(Reçu le 26 juin 1984, révisé le 2 octobre, accepté le 29 octobre 1984)

\begin{abstract}
Résumé. - Nous présentons pour la première fois une étude numérique des modes de vibration pour des amas de percolation constitués de masselottes élastiquement connectées. La densité d'états est caractérisée par une loi de puissance dans le régime de fréquence fracton tandis qu'à plus basse fréquence, on observe un spectre de type Debye. Les états fractals sont faiblement localisés, avec une grande dispersion dans les longueurs de localisation. Les états de basse fréquence correspondent à des états étendus.
\end{abstract}

\begin{abstract}
We present for the first time a numerical study of the vibrational modes of a percolating cluster of mass points connected by elastic bonds. The density of states is characterized by a power law behaviour in the fracton frequency regime, while a transition to a Debye type spectrum occurs at lower frequencies. We also find that the fracton states are weakly localized with a wide range of localization lengths while the states in the low frequency regime are extended.
\end{abstract}

Recently there has been a growing interest in physical problems on fractal structures [1-10]. In particular, much attention has been given to the problem of diffusion of excitations on such structures $[1,4,6,7-10]$ and to the vibrational properties of a system of atoms which has fractal geometry [1-3]. The structure of percolating clusters above the percolation threshold is fractal on a scale smaller than $\xi$ the percolation correlation length, and becomes Euclidean on larger length scales. This geometry is reflected in the properties of the diffusion of excitations in these systems. A characteristic time $\tau$ becomes relevant which corresponds to the diffusion over a distance $\xi$, and which diverges near $p_{\mathrm{c}}$ as $\tau \sim\left(p-p_{\mathrm{c}}\right)^{-d_{\mathrm{w}} v}$ where $d_{\mathrm{w}}=(\mu+2 v-\beta) / v, \mu$ is the percolation conductivity exponent, $v$ is the correlation exponent, and $\beta$ is related to the probability of a site to belong to the percolating cluster. For $t<\tau$ the diffusion is anomalously slow and the diffusion length depends on time as $R \sim t^{\left(1 / d_{w}\right)}$, while at longer times the diffusion is characterized by a finite diffusion coefficient which vanishes at $p=p_{\mathrm{c}}$. Extension of these arguments [1] to the probability of the excitation to be at the origin after time $t,\left\langle P_{0}(t)\right\rangle$ goes as $t^{-\tilde{d} / 2}$ for $t<\tau$ and $t^{-d / 2}$ for $t>\tau$. The parameter $d=2 D / d_{\mathrm{w}}$, where $D$ is the Hausdorff dimension of the percolating clusters, was termed the fracton dimensionality by Alexander and Orbach [1], who also suggested that it has the value of $d=4 / 3$ for percolation fractals in all dimensions. While there is still some question about whether the result $d=4 / 3$ is exact in alf dimensions [11], it is nevertheless very nearly exact. Alexander and Orbach [1] applied these ideas to the vibrational spectrum of a system of atoms for a single percolating cluster with nearest- 
neighbour atoms connected by elastic bonds. The simplest Hamiltonian that yields a non-trivial vibrational spectrum is the Born Hamiltonian [12, 13],

$$
H=\frac{1}{2} \sum_{\mathrm{nn}} K_{i j}\left[\alpha\left(\mathbf{u}_{i}-\mathbf{u}_{j}\right)_{\|}^{2}+\beta\left(\mathbf{u}_{i}-\mathbf{u}_{j}\right)_{\perp}^{2}\right]
$$

where the sum is over all nearest-neighbour pairs. Here $\left(\mathbf{u}_{i}-\mathbf{u}_{j}\right)_{\|}$is the relative displacement in the direction of the bond $(i, j)$ and $\left(\mathbf{u}_{i}-\mathbf{u}_{j}\right)_{\perp}$ is the relative displacement in the perpendicular direction. For random site percolation, $K_{i j}$ is a random variable which is 1 if the sites $i$ and $j$ are both occupied, and 0 otherwise. By changing the parameters $\alpha$ and $\beta$ two extreme cases can be obtained [13] : for $\alpha=1$ and $\beta=0$ the problem reduces to that of a set of mass points connected by springs. For a simple hypercubic lattice the system has a finite fraction of zero frequency modes for $p<1$. For other lattices such as fcc, zero frequency modes will appear for $p<p_{\mathrm{r}}$ where $p_{\mathrm{r}}$ is the rigidity threshold of the lattice at which the macroscopic rigidity of the system vanishes. The value of $p_{\mathrm{r}}$ is considerably higher than $p_{\mathrm{c}}[13,14]$. We analyse here the case where $\alpha=\beta=1$ and $p>p_{\mathrm{c}}$. The system is macroscopically rigid. There are only two zero frequency modes for $p>p_{\mathrm{c}}$, which correspond to rigid translation of the system. The displacements of the mass points are given by

$$
m_{i} \ddot{\mathbf{u}}_{i}=\sum_{j} K_{i j}\left(\mathbf{u}_{i}-\mathbf{u}_{j}\right)
$$

where the sum is over the nearest neighbours of site $i$. In the frequency domain, we have

$$
-m_{i} \omega^{2} \mathbf{u}_{i}^{\omega}=\sum_{j} K_{i j}\left(\mathbf{u}_{i}^{\omega}-\mathbf{u}_{j}^{\omega}\right) .
$$

For site percolation, each occupied site has a mass $m_{i} \equiv 1$. The bonds connecting occupied sites to boundary sites are considered to be severed so that $K_{i j}=0$ if either sites $i$ or $j$ are unoccupied and $K_{i j}=1$ otherwise. Since only sites in the same cluster are connected, each cluster vibrates independently.

The set of equations given by (3) are analogous to the Laplace transformed equations for diffusion on a lattice with site percolation disorder. The local displacements $u_{i}^{\omega}$ correspond to the probability $P_{i}(s)$ of the diffusing particle to be at site $i$. The frequency $s$ in the diffusion case corresponds to $-\omega^{2}$ in the vibrational case. One can then apply the same scaling analysis used for the diffusion case to the present problem. Alexander and Orbach [1] proposed that the fractal geometry of a system is reflected in theinature of the vibrational states. For the vibrational density of states, the fracton dimensionality $\tilde{d}$, plays the role of the regular space dimensionality. In analogy with the diffusion properties, for each value of fraction $p$ of occupied sites $p>p_{\mathrm{c}}$, a crossover frequency $\omega_{\mathrm{c}}$ exists such that for $\omega>\omega_{\mathrm{c}}$ the density of states $N(\omega)$ is fracton-like with $N(\omega) \cong \omega^{d-1}$ while for $\omega<\omega_{\mathrm{c}}$ it is similar to a Debye spectrum, $N(\omega) \cong \omega^{d-1}$. $\omega_{\mathrm{c}}$ vanishes at $p_{\mathrm{c}}$ where the system becomes fractal on all length scales : $\omega_{\mathrm{c}}^{2} \sim\left(p-p_{\mathrm{c}}\right)^{d_{\mathrm{w}} v}$. It was further conjectured that the states in the fracton regime are localized in contrast to the extended Debye like states [1-3].

In this Letter, we present a numerical study of the vibrational modes of a percolating network. The network consist of a single very large cluster which spans the sample (the " infinite » cluster). Our results show that near the percolation threshold the density of states has a fracton behaviour. Above $p_{\mathrm{c}}$ we see a transition from a behaviour which is roughly Debye like at low frequencies to a fracton behaviour at higher frequencies. The transition between the two regimes seems smooth and the density of states does not show any singular behaviour at the crossover region. We have also analysed the spatial extent of the various modes by evaluating the inverse participation ratio. Our results show that, as expected, all the states in the Debye regime are extended, but not 
all the states in the fracton regime are localized. The crossover from extended to localized states is smooth, in constrast to the earlier conjecture by Alexander et al. [3]. We also find some structure in the density of states which is not predicted by either the scaling arguments or the effective medium approximation (EMA) calculations [7, 15, 16].

It is interesting to note that the Hamiltonian given by equation (1) does not represent a unique way to describe the static and elastic dynamic properties of random networks [17]. In a future publication [18] we will present a study of the vibrational properties of random networks based on non-scalar rotational invariant interactions. It was recently shown that this type of Hamiltonian, which may yield a correct description of the mechanical properties of fractal colloidal aggregates, is not related to a simple diffusion problem. However, the present model is important, not only for its relevance to the vibrational modes of some systems but also for its connection to diffusion and conductivity. It is not clear at this stage what is the relative importance of these two classes of interactions [19].

It is interesting to compare the information contained in the density of eigenstates of equation(3) with the information that can be obtained by the simulation of diffusion on percolating clusters [9, 10]. In the latter case, the mean square displacement of the ant from the origin at time $t,\left\langle R^{2}(t)\right\rangle$ and the probability of the diffusing excitation to be at the origin at time $t,\left\langle P_{0}(t)\right\rangle$ are studied. The diffusive properties are determined by the behaviour of the Green function $G_{0}(s)$ for positive small $s$. In this regime the Green function is real, and it is given by the Laplace transform of $\left\langle P_{0}(t)\right\rangle$ [20]. The vibrational density of states is determined by $\operatorname{Im}\left(G_{0}(s)\right)$ at $s=-\omega^{2}$. The singular behaviour of $\operatorname{Im}\left(G_{0}(-z)\right)$ is identical to that of $\operatorname{Re}\left(G_{0}(z)\right)$ for small positive $z$ and this leads to a close relationship between the asymptotic properties of the diffusion at long times, and the density of states of vibrations at low frequency. However, the only information one can obtain from this relationship is the value of the exponent $\tilde{d}$. In order to study any property of the phonon spectrum other than the power law behaviour at the low frequency limit, such as the range of frequencies at which the power law behaviour is valid, the properties of the crossover between the fractal and Debye regimes around $\omega_{\mathrm{c}}$, or the spatial nature of the vibrational states at various frequencies, one has to study the density of phonon states directly. For example, Derrida et al. [15] find that within the EMA, the density of states has a sharp transition between the Debye and fracton region at $\omega_{c}$ in the density of states, which is not reflected in $\left\langle P_{0}(t)\right\rangle$ for $t \cong 1 / \omega_{\mathrm{c}}$.

For the case considered here, the resulting matrix equations given by equation (3) for a cluster of size $N$ decomposes into three $N \times N$ matrices, instead of one $3 N \times 3 N$ matrix. This would not be true if the interactions were either of longer range or contained more general interactions which couple motion parallel and perpendicular to the direction of the spring. We have solved these matrix equations in three dimensions for large, percolating clusters on lattices of size up to $30^{3}$. First, a very large percolating cluster was generated using a growth algorithm [21], thus we consider only the largest cluster in the system. For matrices smaller than $N \cong 2200$, we were able to use standard diagonalization routines [22] to obtain all the eigenvalues of the matrix. For larger systems, we used a recursive technique [23] to calculate the eigenfunctions and eigenvalues in the low frequency region. The recursive technique could be iterated to obtain very accurate eigenvalues and eigenfunctions. We found that the standard diagonalization routines were sufficiently accurate except at low frequency, where they are always supplemented by results using the recursive program. Our results for the density of states for four values of $p$ are presented in figure 1 . These results were obtained by averaging the density of states for 3 percolating clusters for each value of $p$. Though even with such a small number of realizations of a percolation cluster, the statistics are very good and are unchanged with only a single realization. This is the case since we calculate an entire eigenspectrum, which contains a considerable amount of information. The size of the lattice was chosen to give $N$ in the range $1500-2200$. In addition, for $p=0.40$, the low frequency eigenstates were obtained for a $N=9720$ cluster on a $30^{3}$ 

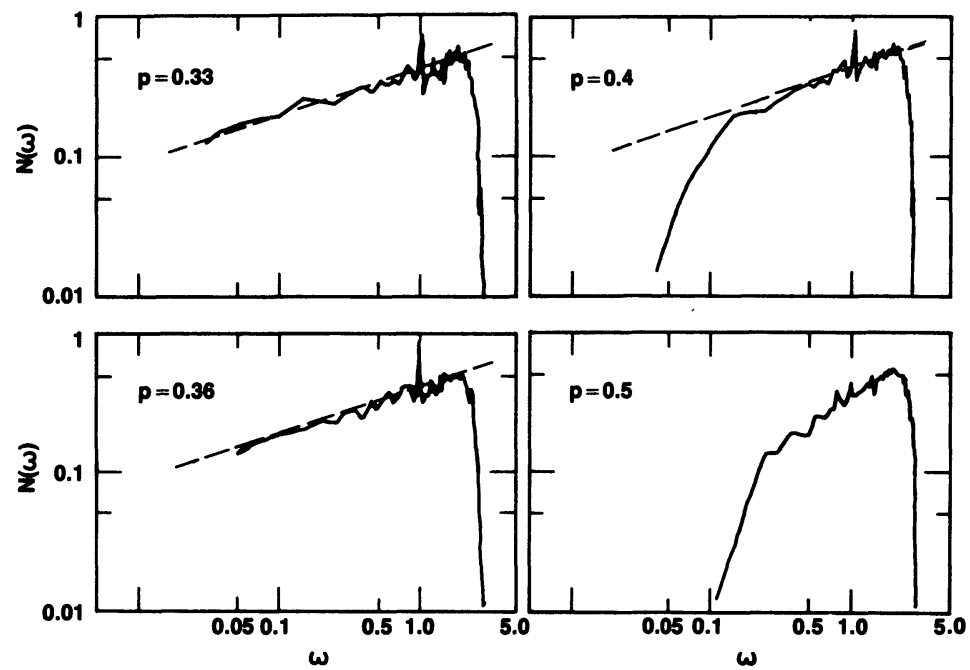

Fig. 1. - Density of states $N(\omega)$ for the percolating clusters versus frequency $\omega$ for four values of the probability $p$. The results are averaged over three clusters of size $N=1388,1435$ and 1489 for $p=0.33$, $N=1575,1646$ and 1687 for $p=0.36$, and $N=2160,2162$ and 2188 for $p=0.40$ on a $18^{3}$ simple cubic lattice. For $p=0.50, N=1334,1344$ and 1340 on a $14^{3}$ lattice. The straight line through the data with a slope of $1 / 3$ for $p=0.33,0.36$ and 0.40 is not a best fit, but only a guide to the eye.

lattice, in order to check the crossover to Debye behaviour for small $\omega$. For $p=0.33, p=0.36$ and $p=0.40$, the density of state is given quite accurately by a power law of the type $N(\omega) \cong \omega^{\tilde{d}-1}$, where $\tilde{d} \cong 4 / 3$, as seen from the straight line through the data which has slope $1 / 3$. Since the site percolation threshold for a simple cubic lattice is $p_{c}=0.3127$, both $p=0.33$ and $p=0.36$ are sufficiently close to this value so that only the fracton regime is observed in the frequency range that we study. For $p=0.40$ and 0.50 two regimes are observed. For low frequencies, $N(\omega)$ increases somewhat more steeply than the $\omega^{2}$ dependence of $N(\omega)$ expected in the Debye regime. A plausible interpretation of this result is that the true Debye regime lies at frequencies lower than our lowest frequency, and that the Debye and fracton regimes are separated by a crossover regime in which the density of state is characterized by a steep increase. This type of behaviour is consistent with the EMA results for the density of states. It is also possible, but less likely, that what is seen at low frequency is part of the Debye regime but the exact value of two for the exponent is not obtained due to finite size effects. At higher frequency, we find $N(\omega) \cong \omega^{\tilde{d}-1}$ for $p=0.40$, while for $p=0.50$ the fracton regime does not seem to be sufficiently wide for such a power law to be apparent. This is not surprising since for $p=0.50$ the correlation length $\xi \sim 3-5$ is not much larger than the size of the unit cell. The crossover frequencies are approximately $\omega_{c}=0.3$ for $p=0.40$ and 0.55 for $p=0.50$. The transition between the two regimes is smooth. In all three cases, $N(\omega)$ falls off very rapidly, for very large frequencies, above the fracton regime.

In order to obtain further insight into the nature of the eigenstates on a percolating cluster, we calculated the low frequency eigenfunctions for a number of samples. For each of these low frequency modes, we computed the inverse participation ratio : $P^{-1}=N \sum_{i} u_{i}^{\omega^{4}} /\left(\sum u_{i}^{\omega^{2}}\right)^{2}$. This quantity is of order unity for extended states, and large for localized states. In figure 2 , we plot $P^{-1}$ versus $\omega$ for a percolating cluster at $p=0.33$ and 0.40 . It is difficult to give a sharp criterion as to which value of $P^{-1}$ corresponds to a transition between localized and extended states. We used the criterion that a mode is localized if $P^{-1}<N^{1 / 2}$, as has been done in numerical 

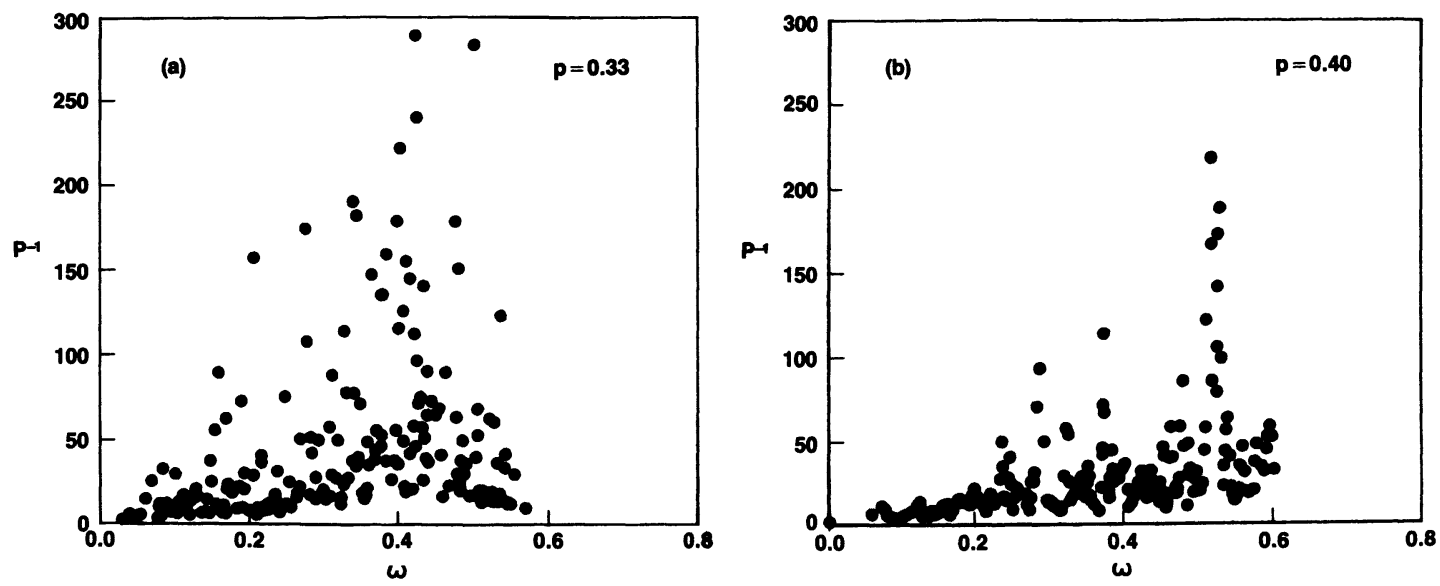

Fig. 2. - Inverse participation ratio for the lowest 200 frequency modes versus frequency for a percolating cluster at $p=0.33$ and $0.40 . N=1489$ for $p=0.33$ and $N=1475$ for $p=0.40$. The range of $\omega$ spans the crossover region for the $p=0.40$ cluster as seen from figure 1 .

studies of electron localization [24]. Applying this criterion to our results (see Fig. 2) leads to the following interesting observation : while all the states in the Debye regime are extended, a considerable fraction of the states in the fracton regime seems to be extended, at least over the size of our system. This suggests that these states are weakly localized with a large localization length. There is no well-defined crossover frequency, which separates localized and extended states. One could argue that this result as well as the absence of any singularity in the density of states at the transition [3] could be caused by the finite size of our lattice. However for $p=0.40$ the correlation length $\xi$ is smaller than the size of the system that we studied.

Another interesting feature of the results shown in figures 2 is the existence of states nearby in frequency with widely varying spatial extents of localization in the fracton regime. This feature of the fracton excitations reflects the absence of any single length scale in the fractal geometry regime of the percolating cluster. It also indicates that these fracton states may coexist with more strongly localized "impurity type " states, corresponding to the highest peaks in $P^{-1}$. Some of these strongly localized states are also responsible for the superimposed peaks in the high frequency regime of the spectrum (Fig. 1). In some cases the local structural element of the cluster corresponding to such a peak can be readily identified. For example, the peak at $\omega=1$ which appears in all the spectra, is related to the vibrations of a single mass point connected to a relatively massive piece of cluster by a single bond. This state, as well as states corresponding to more complex local spatial structures, should be exponentially localized.

To summarize, we have demonstrated the existence of fracton vibrational excitations on percolating clusters. The fracton states are weakly localized and possess no single localization length, reflecting the underlying self-similar structure. The density of states obeys a power law behaviour in which the fracton dimensionality plays the role of the Euclidean dimensionality for regular systems. Below a critical frequency which depends on $\left(p-p_{c}\right)$ the states are extended and lose their fracton nature.

\section{Acknowledgments.}

We thank C. M. Soukoulis and Y. Kantor for helpful discussions. 


\section{References}

[1] Alexander, S. and Orbach, R., J. Physique Lett. 43 (1982) L-625.

[2] Shender, E. F., J. Phys. C 9 (1976) L-309.

[3] Alexander, S., Laermans, C., Orbach, R. and Rosenberg, H. M., Phys. Rev. B 28 (1983) 4615 ; AleXander, S., in Ann. Israel Phys. Soc. 5, " Percolation Structures and Processes ", ed. by G. Deutscher, R. Zallen and J. Adler (Adam Hilger, Bristol) 1983.

[4] Rammal, R. and Toulouse, G., J. Physique Lett. 44 (1983) L-13.

[5] Domany, E., Alexander, S., Bensimon, D. and Kadanoff, L. P., Phys. Rev. B 28 (1983) 3110.

[6] Gefen, Y., Aharony, A. and AleXander, S., Phys. Rev. Lett. 50 (1983) 77.

[7] Webman, I., Phys. Rev. Lett. 47 (1981) 1496.

[8] De GenNes, P. G., La Recherche 7 (1976) 919.

[9] Pandey, R. and Stauffer, D., Phys. Rev. Lett. 51 (1983) 527.

[10] Ben Avraham, D. and Havlin, S., J. Phys. A 15 (1982) L-691.

[11] Aharony, A. and Stauffer, D., Phys. Rev. Lett. 52 (1984) 2368 ;

Hong, D. C., Havlin, S., Herrmann, H. J. and Stanley, H. E. (preprint);

LOBB, C. J. and FraNK, D. J. (preprint);

ZABOLITZKY, J. G. (preprint);

Herrmann, H. J., Derrida, B. and Vannimenus, J. (preprint).

[12] Born, M. and Huang, K., Dynamical Theory of Crystal Lattices, Ch. 5 (Oxford, New York) 1954.

[13] Feng, S. and Sen, P. N., Phys. Rev. Lett. 52 (1984) 216.

[14] THORPE, M., J. Cryst. Solids 57 (1983) 355.

[15] Derrida, B., Orbach, R. and Kin-Wah Yu, Phys. Rev. B 29 (1984) 6645.

[16] Entin-Wohlman, O., Alexander, S., Orbach, R. and Kin-Wah Yu, Phys. Rev. B 29 (1984) 4588.

[17] Kantor, Y. and Webman, I., Phys. Rev. Lett. 52 (1984) 1891.

[18] Webman, I. and Grest, G. S., to be published.

[19] Alexander, S., to be published.

[20] Economu, E. N., Green's Functions in Quantum Physics (Springer, Heidelberg) 1983.

[21] Leath, P. L., Phys. Rev. B 14 (1976) 5046.

[22] Smith, B. T. et al., Matrix Eigensystem Routines (Springer, Berlin) 1974. IMSL routine EIGRS for real symmetric matrices.

[23] Atomic Energy Research Establishment (Harwell) Program EA12A for sparse, real symmetric matrices.

[24] D. Thouless, in Ill-Condensed Matter, edited by R. Balian, R. Maynard and G. Toulouse (NorthHolland, Amsterdam) 1979. 